\title{
Association of long-chain acyl-coenzyme A synthetase 5 expression in human breast cancer by estrogen receptor status and its clinical significance
}

\author{
MENG-CHI YEN $^{1 *}$, JUNG-YU KAN ${ }^{2,3 *}$, CHIA-JUNG HSIEH ${ }^{4,5}$, \\ PO-LIN KUO ${ }^{2}$, MING-FENG HOU ${ }^{2,3}$ and YA-LING HSU ${ }^{4}$ \\ ${ }^{1}$ Department of Emergency Medicine, Kaohsiung Medical University Hospital, Kaohsiung 807; \\ ${ }^{2}$ Graduate Institute of Clinical Medicine, College of Medicine, Kaohsiung Medical University, Kaohsiung 807; \\ ${ }^{3}$ Department of Breast Surgery, Kaohsiung Medical University Hospital, Kaohsiung 807; \\ ${ }^{4}$ Graduate Institute of Medicine, College of Medicine, Kaohsiung Medical University, Kaohsiung 807; \\ ${ }^{5}$ Department of Chinese Medicine, Kaohsiung Medical University Hospital, Kaohsiung 807, Taiwan, R.O.C.
}

Received February 6, 2017; Accepted April 21, 2017

DOI: $10.3892 /$ or.2017.5610

\begin{abstract}
The lipid metabolic enzymes are considered candidate therapeutic targets for breast cancer. Long-chain acyl-coenzyme A (CoA) synthase (ACSL) is one of lipid metabolic enzymes and converts free-fatty acid to fatty acid-CoA. Five ACSL isoforms including ACSL1, ACSL3, ACSL4, ACSL5 and ACSL6 are identified in human. High ACSL4 expression has been observed in aggressive breast cancer phenotype. However, the role of other isoforms is still little-known. We therefore, analyzed the expression of ACSL isoforms in each subtype of breast cancer within METABRIC dataset and cancer cell line encyclopedia dataset. The expression levels of ACSL1, ACSL4 and ACSL5 in estrogen receptor (ER)-negative group were higher than that in ER-positive group. Similar expression pattern was detected among breast cancer cell lines MCF-7 (ER-positive) and MDA-MB-231 (ER-negative). Treatment of ACSL inhibitor triacsin C which inhibited enzyme activity of ACSL 1, 3, 4 and 5 suppressed cell growth of MCF-7 and MDA-MB-231. Our results further showed that high ACSL5 expression was associated with good prognosis in patients with both ER-positive and ER-negative breast cancer through KM plotter analysis. These results
\end{abstract}

Correspondence to: Professor Ming-Feng Hou, Graduate Institute of Clinical Medicine, College of Medicine, Kaohsiung Medical University, No. 100, Shih-Chuan 1st Road, Kaohsiung 807, Taiwan, R.O.C.

E-mail: mifeho@kmu.edu.tw

Professor Ya-Ling Hsu, Graduate Institute of Medicine, College of Medicine, Kaohsiung Medical University, No. 100, Shih-Chuan 1st Road, Kaohsiung 807, Taiwan, R.O.C.

E-mail: hsuyl326@gmail.com

${ }^{*}$ Contributed equally

Key words: long-chain acyl-CoA synthetase, ACSL1, ACSL4, ACSL5, breast cancer, estrogen receptor suggest that ACSL1, ACSL4 and ACSL5 expression is regulated by ER signaling pathways and ACSL5 is a potential novel biomarker for predicting prognosis of breast cancer patients.

\section{Introduction}

Dysregulation of metabolic pathways, including regulation of glucose transporter, tricarboxylic acid cycle (TCA cycle), pentose phosphate pathway and mitochondria respiratory chain are observed in many types of cancers (1). In addition, amino acid glycine, serine and glutamine metabolic pathways play important roles in cancers $(2,3)$. Recent evidence indicates that significant different lipid metabolites and expression of lipid metabolic enzymes are detected in cancer. These lipid metabolites are associated with cell proliferation, cellular membrane synthesis and signaling molecules (4-6). Some of the dysregulated metabolic enzymes, such as the glucose transporter 1 (GLUT1), hexokinase 2, lactate dehydrogenase A, glutaminase and fatty acid synthase have been demonstrated to be novel therapeutic targets of cancers (7). The metabolomics in serum or plasma will be novel diagnostic approach in clinic (8). Therefore, investigating the correlation between metabolites, metabolic enzymes and cancer is a critical issue.

The latest statistics reveal that breast cancer is still one of the most common cancer types and leading cause of cancer death (9). The molecular subtypes of breast cancer could be divided into four types: luminal A, luminal B, triplenegative/basal-like and HER2 type. Luminal type tends to express estrogen receptor (ER), HER2 type is HER2 (human epidermal growth factor receptor 2) positive and progesterone receptor (PR), ER and HER2 expression is negative in triplenegative/basal-like type $(10,11)$. Various studies have shown that the expression of metabolic enzymes is associated with ER, PR and HER2. Triple-negative breast cancer cells express the highest level of GLUT1 compared to other types of breast cancer cells (12). Immunohistochemistry assay shows that HER2 positive and triple-negative breast cancer cells express relatively high level of glutamate-metabolic enzymes (13). The 
evidence suggests that the expression of ER, PR and HER2 is associated with various metabolic enzymes in breast cancer.

Most breast cells acquire fatty acids from circulation system. However, breast cancer cells synthesize fatty acids for structured lipid synthesis (7). Fatty acid synthase (FAS) is an important enzyme in lipid synthesis pathway. High FAS expression is usually observed in HER2-positive breast cancer and the HER2-FAS-related signaling pathway might promote proliferation, metastasis and chemotherapy resistance (14-16). Blockage of FAS induces apoptosis in breast cancer cells (17). Combination of trastuzumab (monoclonal antibody against HER2) and FAS inhibitor results in re-sensitization with trastuzumab in the trastuzumab-resistant breast cancer cells (18). Synergistic therapeutic effect is observed after combination of FAS inhibitors and other chemotherapies $(19,20)$. Therefore, blockage of lipid metabolic enzymes might be a novel strategy for breast cancer treatment.

In mammalian cells, the conversion between free-fatty acid and fatty acid-CoA are catalyzed by a fatty acyl-CoA synthase (ACS) which is classified by catalyzing substrates. A free-fatty acid containing 14-20 carbons is the substrate of long-chain acyl-CoA synthetases (ACSL) (21). The five isoforms of ACSL include ACSL1, ACSL3, ACSL4, ACSL5 and ACSL6. All enzymes have individual functions in substrate preference and tissue specificity (22). Based on the sequence homology, the five ACSL isoforms are divided into two groups: one is composed of ACSL1, ACSL5, ACSL6 and the other ACSL3 and ACSL4 (23). ACSL family convert long-chain fatty acid to fatty-acid-CoA which is essential component for $\beta$-oxidation which was suggested to promote oncogenesis in breast cancer (24,25). A study indicates that the ER expression level is negatively associated with ACSL4 expression through 10 published mRNA array datasets in breast cancer cell lines (26). In addition, ACSL4 is considered a biomarker for breast cancer and is associated with aggressive breast cancer type (27). However, the association between other ACSL isoforms and molecular subtypes of breast cancers is poorly known. In the present study, we aimed to investigate this issue in each breast cancer subtype from gene expression datasets and in breast cancer cell lines.

\section{Materials and methods}

Bioinformatics analysis: $m R N A$ expression levels. The clinical data of breast cancer samples and mRNA expression levels of ACSL1, ACSL3, ACSL4, ACSL5 and ACSL6 was downloaded as Z-Scores from the cBioPortal (http://www. cbioportal.org, Breast cancer, Metabric, Nature 2012 \& Nat Commun 2016, 2509 samples, Version 1.3.3) (28,29). The expression levels of ACSL isoforms were analyzed through Oncomine Research Edition which includes Kao cohort (30), Hatzis cohort (31), Minn cohort (32), Miyake cohort (33), van de Vijver cohort (34), and Wang cohort (35) (Thermo Fisher Scientific; http://www.oncomine.org, v4.5) and the Cancer Cell Line Encyclopedia (CCLE) database (https:// portals.broadinstitute.org/ccle/home) (36). The heatmap was draw by GENE-E software.

Assessment of the patient survival rate. The survival analysis in breast cancer patients with different expression levels of
ACSL isoforms were performed through the KM-Plotter database (37). The prognostic value of each gene was analyzed by splitting patient samples into two groups by median, after the subtype of breast cancer was restricted to different ER status. The relapse-free survival rate was analyzed (2016.10.13 update, the breast cancer database includes 5,143 samples).

Cell culture. Normal breast epithelial cell line H184B5F5/M10 were obtained from the Bioresource Collection and Research Center (Hsinchu, Taiwan). Human breast cancer cell lines MCF-7 and MDA-MB-231 were kindly provided by Professor Ming-Derg Lai in National Cheng-Kung University (38). Cells were maintained in recommended media (H184B5F5/M10 was in alpha-Minimum Essential Medium (MEM). MCF-7 and MDA-MB-231 cells were in defined MEM (Lonza, Walkersville, MD, USA). Both media were supplemented with $10 \%$ fetal bovine serum (FBS) and penicillin/streptomycin (100 U/0.1 mg/ml) (Life Technologies, Inc., Grand Island, NY, USA).

Quantitative PCR. Total RNA of MCF-7, MDA-MB-231 and H184B5F5/M10 was extracted using TRIzol (Invitrogen, Carlsbad, CA, USA). Complementary DNA was produced from 500 ng total RNA was using a PrimeScript RT reagent kit (Clontech Laboratories, Inc., Kusatsu, Japan). The levels of ACSL isoforms were determined on a Real-Time PCR system (StepOne Plus Real-Time PCT System; Applied Biosystems, Foster City, CA, USA) using Fast SYBR-Green Master Mix (Applied Biosystems). The primers of ACSL isoforms were obtained from a previous report (39) and glyceraldehyde3-phosphate dehydrogenase (GAPDH) were 5'-GAGTCAA CGGATTTGGTCGT-3' and 5'-TTGATTTTGGAGGGATCT CG-3'. Relative mRNA expression levels of ACSL isoforms were normalized to the expression level of GAPDH and calculated by $2^{-\Delta \Delta \mathrm{Ct}}$ method.

Western blot analysis. Cells were lysed in RIPA lysis buffer (Millipore, Billerica, MA, USA) and protein concentration was quantitated by BCA protein assay kit (Millipore). Each protein was detected by using primary antibody (anti-ACSL antibody, \#4047; Cell Signalling Technology, Danvers, MA, USA), anti-ACSL4 (ab155282; Abcam, Cambridge, UK), antiACSL5 (ab57210; Abcam) and GAPDH (MAB374; Millipore). The results were analyzed on an imaging capture system (Alpha Innovation).

Evaluation of proliferation rate. For cell proliferation measurement, WST-1 (Clontech Laboratories) was used and then $2 \times 10^{3}$ MCF-7, MDA-MB-231 and H184B5F5/M10 cells were seeded in 96-well plates with different concentration of ACSL inhibitors including triacsin C (Abcam), rosiglitazone (Sigma-Aldrich) and 2-fluoropalmitic acid (Cayman Chemical, Ann Arbor, MI, USA) in 0.8\% dimethyl sulfoxide (DMSO). The proliferation rate was determined at wavelength $450 \mathrm{~nm}$ on a microplate spectrophotometer (PowerWave X340; BioTek Instruments, Inc., Winooski, VT, USA) after 48 h of treatments.

Statistical analysis. All graphs were generated by GraphPad Prism 7 (GraphPad Software, Inc., San Diego, CA, USA). 


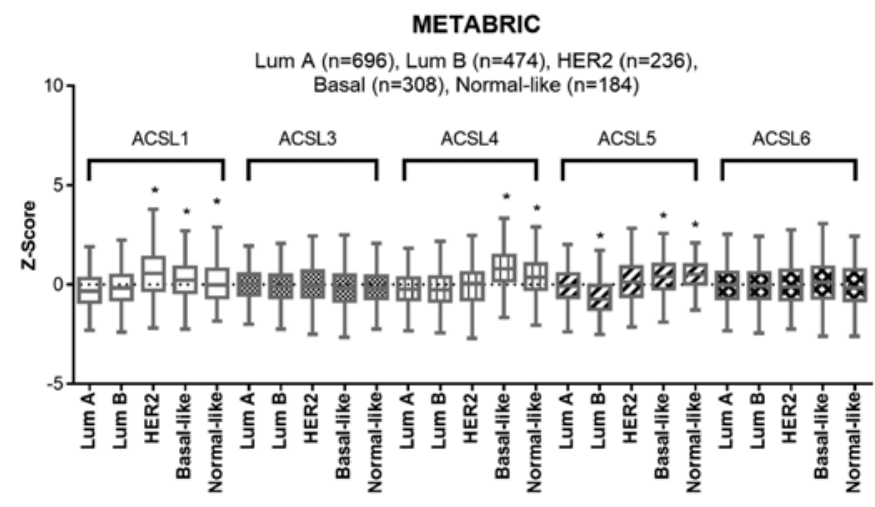

Figure 1. Expression levels of ACSL isoforms in five molecular subtypes of breast cancer on METABRIC dataset. Boxplot shows the Z-Score of ACSL1, ACSL3, ACSL4, ACSL5 and ACSL6 expression. ${ }^{*} \mathrm{P}<0.001$ indicates difference in mRNA levels of each ACSL isoform among five subtypes when compared with subtype Lum A. Lum A and Lum B, luminal A and luminal B.

Student's t-test or one-way ANOVA was used for analysis of difference between two groups and three groups, respectively. $\mathrm{P}<0.05$ was considered to indicate a statistically significant difference.

\section{Results}

Low expression levels of ACSL1 and ACSL5 is observed in luminal A subtype in the METABRIC dataset. We analyzed the expression levels of five ACSL isoforms among five subtypes of breast cancer in METABRIC dataset (Fig. 1). No significant difference was detected in the expression levels of ACSL3 and ACSL6 among each subtype of breast cancer. Compared to luminal A subtype, higher mRNA levels of ACSL1, ACSL4 and ACSL5 were shown in basal-like and normal-like subtypes. In addition, relatively high mRNA level of ACSL1 was observed in HER2 subtype. Since high ER/PR expression and low ER/ PR expression is, respectively, a characteristic of luminal A subtype and basal-like subtype, the results may imply that high expression levels of ACSL1, ACSL4 and ACSL5 are associated with low ER/PR expression. In addition, ACSL1 expression is associated with HER2 expression.

The expression levels of ACSL1 and ACSL5 is associated with $E R$ and PR expression in breast cancer cell lines. A previous report indicates that ACSL4 expression is negatively associated with sex steroid hormone receptor in breast cancer (26). To further investigate the relationship between ACSL1, ACSL5 and ER/PR and HER2 status, we analyzed it in human breast cancer cell lines. In Fig. 2A, the mRNA expression of ACSL1, ACSL4 and ACSL5 was analyzed through different probes in several breast cancer cell lines within the Cancer Cell Line Encyclopedia (CCLE) database. The status of ER and HER2 is based on a previous report (40). The result revealed that the lowest expression of ACSL1, ACSL4 and ACSL5 was in MCF-7 cells (luminal A) compared to other cell lines. However, the expression pattern of ACSL1, ACSL4 and ACSL5 is not associated with the HER2 and basal-like subtypes. It suggests that all three ACSL isoforms are associated with ER/PR. We further determine the mRNA and protein expression levels of ACSL isoforms in MCF-7, MDA-MB-231, and H184B5F5/
A
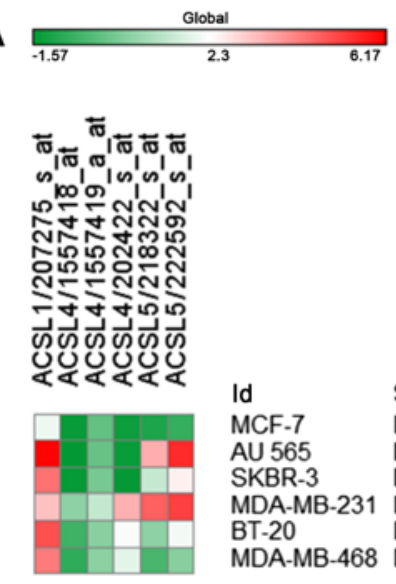

$\begin{array}{ll}\text { Id } & \text { Subtypes } \\ \text { MCF-7 } & \text { Luminal A } \\ \text { AU 565 } & \text { HER2 } \\ \text { SKBR-3 } & \text { HER2 } \\ \text { MDA-MB-231 } & \text { Basal } \\ \text { BT-20 } & \text { Basal } \\ \text { MDA-MB-468 } & \text { Basal }\end{array}$

Receptor expression

ER+/PR+, HER2-

ER-/PR-, HER2+

ER-/PR-, HER2+

ER-/PR-, HER2-

ER-/PR-, HER2

ER-/PR-, HER2-

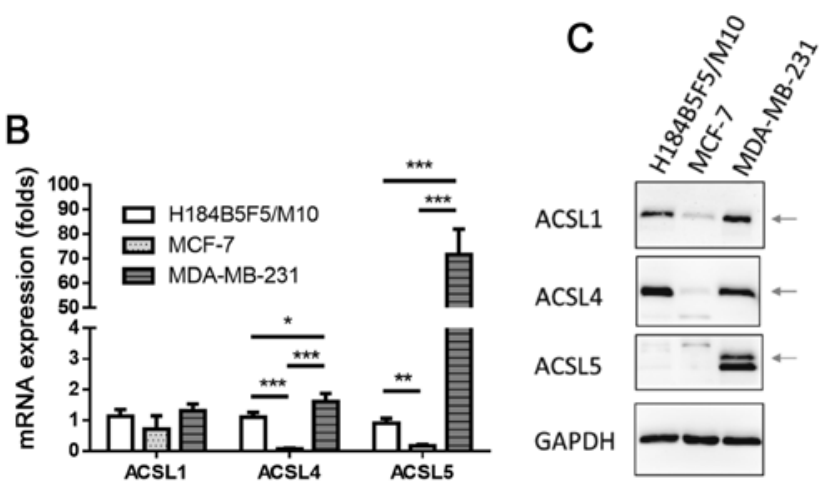

Figure 2. Expression levels of ACSL1, ACSL4 and ACSL5 in human breast cancer cell lines. (A) Heatmap displaying mRNA expression of ACSL isoforms in breast cancer cell lines with different subtypes through CCLE database. (B) mRNA expression and (C) protein expression of ACSL1, ACSL4 and ACSL5 in H184B5F5/M10, MCF-7 and MDA-MB-231. The error bars represent SD. The statistical difference between each group was examined with one-way ANOVA test $\left({ }^{*} \mathrm{P}<0.05,{ }^{* * *} \mathrm{P}<0.01,{ }^{* * * *} \mathrm{P}<0.001\right)$.

M10 which is a normal breast epithelial cell line. In Fig. 2B and $\mathrm{C}$, relatively high expression of ACSL isoforms was observed in MDA-MB-231 cells. It might suggest that ACSL1, ACSL4 and ACSL5 expression is associated with ER/PR expression in breast cancer. Notably, similar expression level of ACSL1 and ACSL4 was detected between H184B5F5/ M10 and MDA-MB-231 (Fig. 3C). The mRNA expression of ACSL5 in H184B5F5/M10 was higher than MCF-7 (Fig. 3B).

Investigating ACSL1 and ACSL5 expression in patients with different ER status within public microarray datasets. To further determine whether ACSL1 and ACSL5 expression is associated with ER expression, we analyzed it within six microarray datasets including Kao cohort (30), Hatzis cohort (31), Minn cohort (32), Miyake cohort (33), van de Vijver cohort (34) and Wang cohort (35). In Fig. 3A-F, ACSL1 levels in ER-negative group was higher than that in ER-positive group. In addition, higher levels of ACSL4 and ACSL5 was respectively observed in Fig. 3A-E and 3B-F. The evidence suggests the ER status is an important factor to regulate ACSL1, ACSL4 and ACSL5 expression.

Investigation of ACSL1, ACSL4 and ACSL5 as therapeutic targets of breast cancer. Previous reports indicate that inhibition of FAS is a strategy to treat breast cancer (17-19). 
A
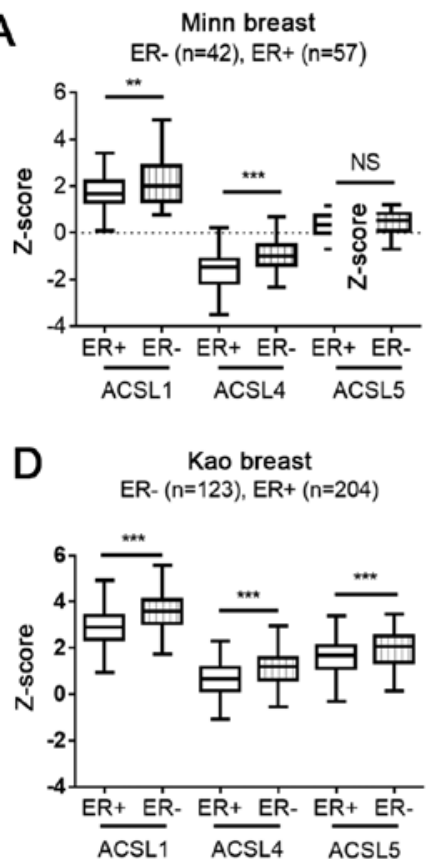

B
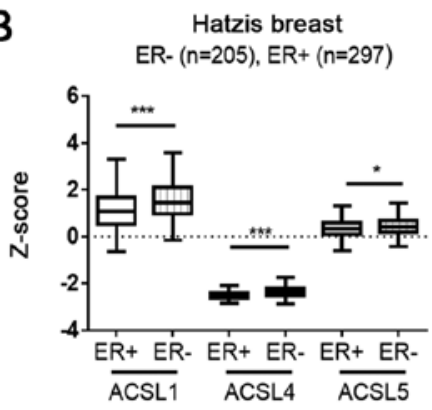

$\mathrm{E}$

van de Vijver breast
ER- $(n=69), E R+(n=226)$

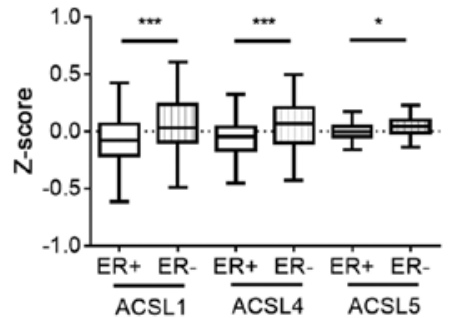

C

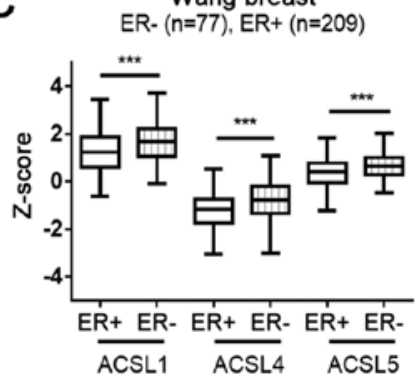

$\mathrm{F}$
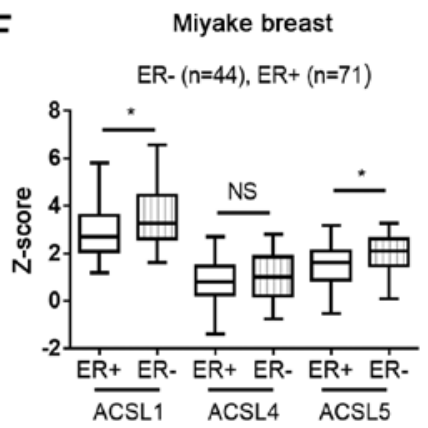

Figure 3. Expression levels of ACSL1, ACSL4 and ACSL5 in patients with different ER status through six microarray datasets including (A) Minn breast, (B) Hatzis breast, (C) Wang breast, (D) Kao breast, (E) van de Vinver breast and (F) Miyake breast. The ER status and expression of three ACSL genes of each dataset were adapted from Oncomine database. The number in parentheses indicate the number of patients. The statistical difference between ER-positive and ER-negative group was examined with the Student's t-test ( ${ }^{*} \mathrm{P}<0.05,{ }^{* * *} \mathrm{P}<0.001 ;$ ns, no significant difference).
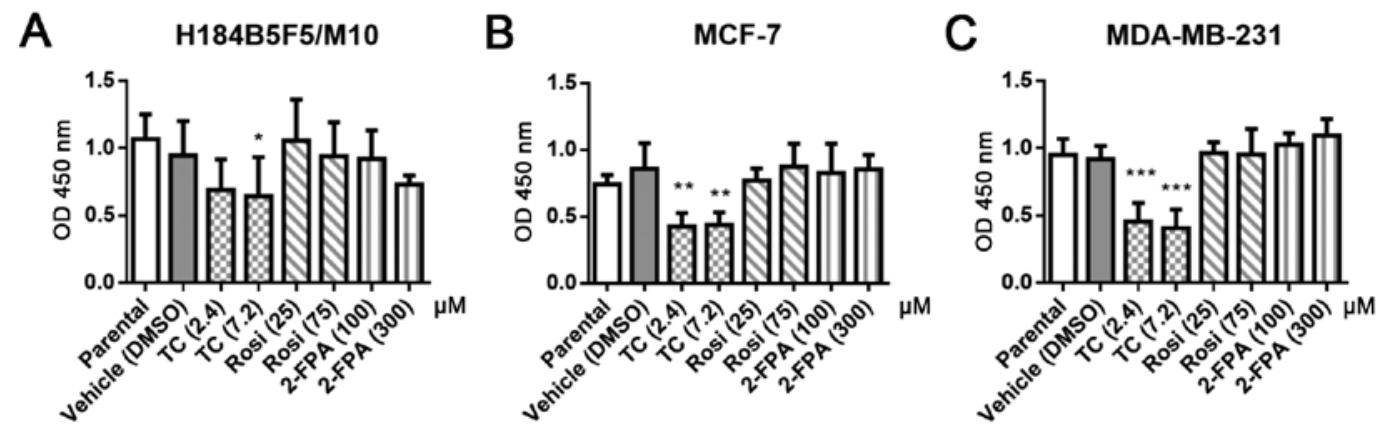

Figure 4. Effect of ACSL inhibitors on cell growth. Cells $\left(2 \times 10^{3}\right)$ were plated and then incubated with the ACSL inhibitors triacsin C, rosiglitazone (Rosi), and 2-fluoropalmitic acid (2-FPA) for $48 \mathrm{~h}$. The cell growth of (A) H184B5F5/M10, (B) MCF-7 and (C) MDA-MB-231 was evaluated by WST-1 assay. The statistical difference between parental and each group was examined with one-way ANOVA test $\left({ }^{*} \mathrm{P}<0.05,{ }^{* *} \mathrm{P}<0.01,{ }^{* * *} \mathrm{P}<0.001\right)$.

We therefore investigated whether ACSL inhibitors resulted in growth inhibition in the ER-positive cell line MCF-7, ER-negative cell line MDA-MB-231 and the normal breast epithelial cell line H184B5F5/M10. Three ACSL inhibitors were chosen. Triacsin $\mathrm{C}$ is an analog of a polyunsaturated fatty acid and competitively inhibits enzyme ACSL 1, 3, 4 and $5(41,42)$. 2-Fluoropalmitic acid is an analog of palmitic acid and a competitive inhibitor of ACSL (43). Rosiglitazone is an agonist of peroxisome proliferator-activated receptor gamma (PPAR- $\gamma$ ) and selectively suppresses ACSL4 activity over other ACSL isoforms (44). Our results revealed that 2 -fluoropalmitic acid and rosiglitazone did not affect cell growth (Fig. 4). In contrast, the growth of all three cell lines, including H184B5F5/M10, was inhibited by triacsin C treatment. It might imply blockage of ACSL activity at an appropriate concentration that may be a strategy to inhibit breast cell growth.
Investigation of ACSL1, ACSL4 and ACSL5 could serve as markers for predicting the survival of patients with breast cancer. Since high expression levels of ACSL1, ACSL4 and ACSL5 was observed in ER-negative breast cancer patients, we further investigated whether expression levels of ACSL1, ACSL4 and ACSL5 were associated with survival of patients with different ER status in breast cancer. Our results show that the expression of ACSL1 and ACSL4 was not significantly associated with survival rate. In contrast, ACSL5 was significantly associated with good survival in all the patients (Fig. 5). It suggests ACSL5 is a potential novel biomarker for predicting prognosis of breast cancer patients.

\section{Discussion}

Recently, a study which use a systematic analysis through public microarray datasets indicated that different types of 

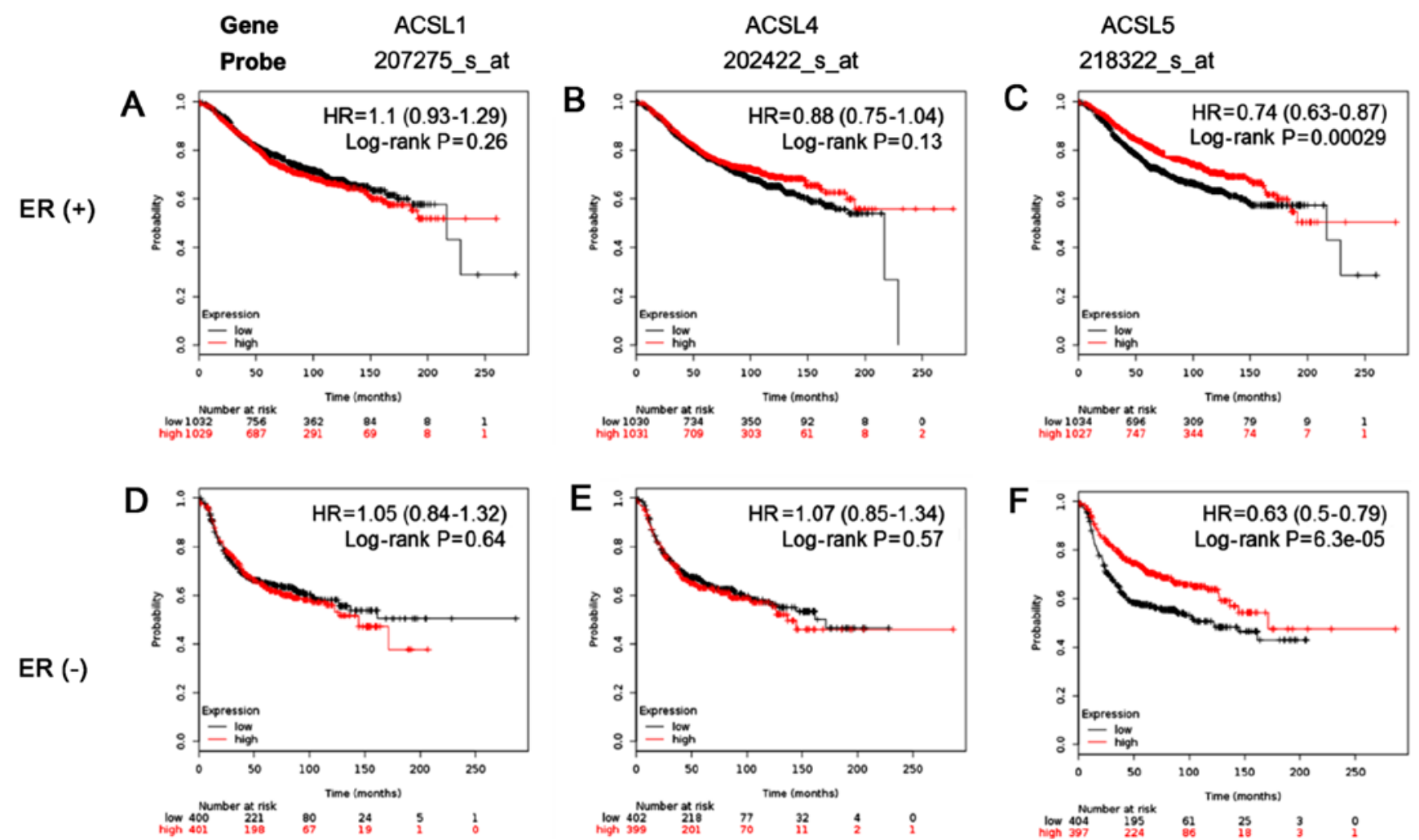

Figure 5. Evaluation of survival curve comparing the patients with high (red) and low (black) ACSL expression from KM plotter database. The survival curve of ER-positive patients with (A) ACSL1, (B) ACSL4 and (C) ACSL5 expression and ER negative patients with (D) ACSL1, (E) ACSL4 and (F) ACSL5 are shown. The hazard ratio (HR) and log-rank P-value are in each figure.

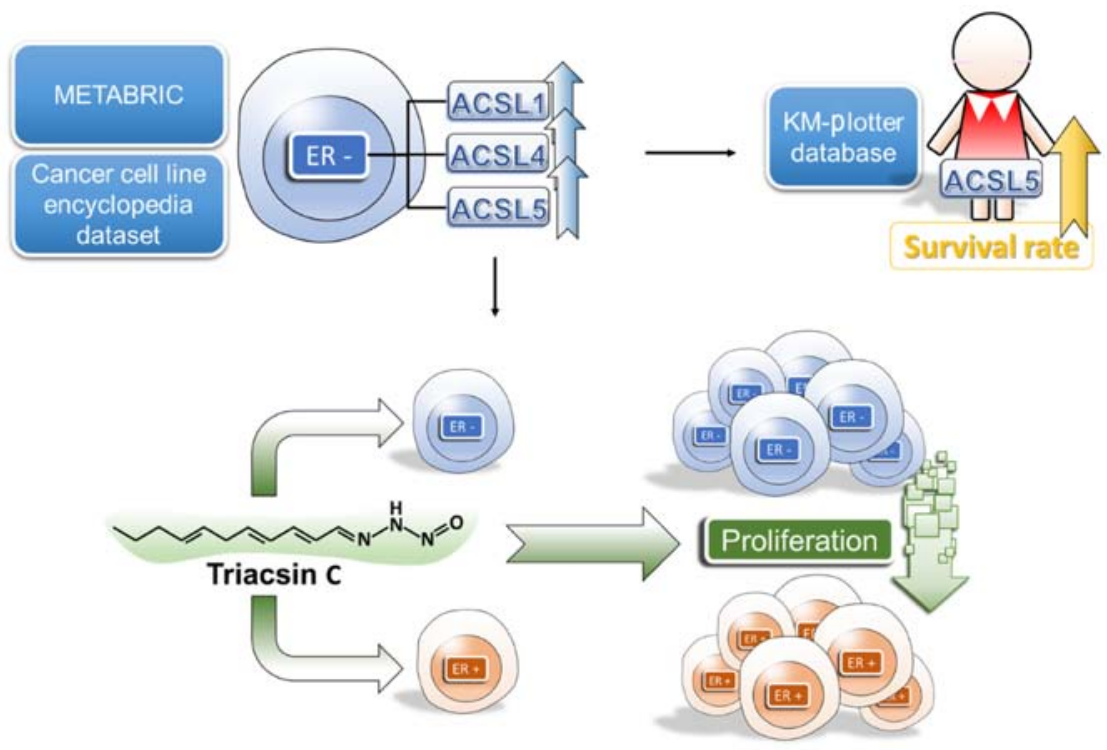

Figure 6. Summary of ACSL isoforms in breast cancer.

ACSL isoforms reveal distinct function in different types of cancers, including breast cancer (45). The present study shows that high ACSL1 expression is correlated with poor overall survival rate and high expression levels of ACSL4 and ACSL5 are correlated to good overall survival rate in breast cancer (45). However, the function of ACSL isoforms were not investigated in different subtypes of breast cancer and the survival analysis was performed in a relatively small cohort.
In this study, we found that the ACSL1, ACSL4 and ACSL5 expression was negatively associated with ER expression in breast cancer patients in large cohorts. Similar expression pattern was detected in breast cancer cell lines. Treatment of ACSL inhibitor triacsin $\mathrm{C}$ inhibited cell proliferation in H184B5F5/M10, MCF-7 and MDA-MB-231 cells. In addition, only ACSL5 could be a potential marker for good survival of breast cancer patients. 
The role of ACSL4 has been investigated in several studies. A report indicates that overexpression of ACSL4 in MCF-7 which expresses low endogenous ACSL4 enhances the ability of cell growth, invasion, anchorage-independent growth in vitro and tumor growth in nude mice (27). However, another study demonstrated that silencing ACSL4 expression in MDA-MB-231 did not affect growth rate, but MDA-MB231 cells sensitize triacsin $C$ treatment (26). In addition, low dose $(<100 \mathrm{mM})$ of ACSL4 inhibitor rosiglitazone did not significantly decrease cell viability in MDA-MB-231 and in MDA-MB-231 xenograft model $(46,47)$. The growth inhibitory effect of high-dose rosiglitazone might be through PPAR- $\gamma$ but not ACSL4 pathway $(44,47)$. Similar results were observed in the present study. The evidence suggests that ACSL4 is not a critical enzyme to increase cell growth and viability in ER-negative breast cancer. On the other hand, a recent study demonstrates that ACSL4-silencing breast cancer cells resist ferroptosis and the w6 fatty acid acids are enriched in cellular membrane under ferroptosis stimulation (48). The mammalian target of rapamycin (mTOR) signaling pathway is regulated by ACSL4 in breast cancer cells (46). ACSL4 involves in deiminase isoform 2-mediated oncogenic pathway in an ER-positive MCF-7 breast cancer cell line (49). The physiological role and regulatory mechanism of ACSL4 needs to be investigated in the future.

The role of ACSL1 and ACSL5 is little-known in breast cancer. The ACSL1 and ACSL5 are in the same group and ACSL4 is another group based on their sequence homology (23). Previous studies have shown that substrate preference of ACSL1 is unsaturated fatty acids oleate (18 carbons) and linoeate (18 carbons) and ACSL5 is palmitic acid (16 carbons), palmitoleic acid (16 carbons), oleic acid (18 carbons) and linoleic acid (18 carbons). Besides, both enzymes are detected in nucleus and mitochondria $(50,51)$. Although ACSL1 and ACSL5 have similar substrate preference and subcellular location, only ACSL5 is associated with survival of patients with ER-positive and ER-negative breast cancer. In the present study, mRNA and protein expression of ACSL1, ACSL4 and ACSL5 in H184B5F5/M10 was higher than that in MCF-7 cells (Fig. 2B and C). We therefore, suppose that the ACSL5 function could be compensated in high ER expression breast cancer, such as Luminal A subtype. Knockdown of ACSL5 in hepatocytes decrease triglyceride synthesis (52). In addition, overexpression of ACSL5 induces neosynthesis of ceramide which is a signaling molecule in the apoptosis pathway (53). The correlation between ER signaling pathways and lipid metabolites should be investigated in breast cancer in further studies.

Targeting fatty acid synthesis is a strategy for cancer treatment. Blockage of FAS enzyme activity-mediated de novo fatty acid synthesis shows antitumor potential in multiple types of cancer (17-20). However, potential side-effects of FAS inhibition is still a concern (54). In our results (Fig. 4) and a previous study (26), triacsin $\mathrm{C}$ is a relatively potent inhibitor to induce apoptosis in breast cancer cells in comparison with other inhibitors. Triacsin $\mathrm{C}$ but not 2-fluoropalmitic acid and rosiglitazone inhibits de novo synthesis of triacylglycerol, diacylglycerol and cholesterol esters and synthesis of phospholipid (55). These results imply that ACSL metabolic products within de novo synthesis pathway are important for prolifera- tion of breast cancer. Although ACSL isoforms might serve as alternative cancer therapeutic targets in process of de novo fatty acid synthesis, high-dose triacsin C (7.2 mM) inhibits growth in normal breast cells (Fig. 4). Low-dose triacsin C might be suitable for breast cancer treatment. However, current evidence could not provide a specific ACSL isoform as the best target for breast cancer treatment.

Estrogen affects reactive oxygen species production in mitochondria in breast cancer (56). In addition, ER $\alpha$ and ER $\beta$ are found in mitochondria and ER $\beta$ interacts with a mitochondrial protein HADHB which is required for $\beta$-oxidation in breast cancer (57). $\beta$-oxidation is reported to promote oncogenesis in breast cancer $(24,25)$. In addition, ACSL1, ACSL4 and ACSL5 are observed in mitochondria and cytosol and the metabolic of ACSL family is essential for $\beta$-oxidation. We suppose that the interaction of ACSL1, ACSL4, ACSL5 and ER in mitochondria might play an important role in development of breast cancer.

In summary, our results have shown that the high expression of ACSL1, ACSL4 and ACSL5 is associated with ER-negative breast cancer. Inhibition of ACSL activity through low-dose triacsin $\mathrm{C}$ might be a strategy to suppress growth in breast cancer cell. Furthermore, our results suggest that high ACSL5 expression is associated with good prognosis in patients with breast cancer (Fig. 6).

\section{Acknowledgements}

The present study was supported by grants from the Ministry of Science and Technology (MOST 105-2314-B-037-037-MY3; MOST 104-2314-B-037-053-MY4; MOST 103-2320-B-037006-MY3), the 'KMU-KMUH Co-Project of Key Research' (grant no. KMU-DK 105002 from Kaohsiung Medical University) and the Kaohsiung Medical University Hospital Research Foundation (KMUH104-4M24; KMUH104-4M56).

\section{References}

1. Chen JQ and Russo J: Dysregulation of glucose transport, glycolysis, TCA cycle and glutaminolysis by oncogenes and tumor suppressors in cancer cells. Biochim Biophys Acta 1826: 370-384, 2012.

2. Locasale JW: Serine, glycine and one-carbon units: Cancer metabolism in full circle. Nat Rev Cancer 13: 572-583, 2013.

3. Hensley CT, Wasti AT and DeBerardinis RJ: Glutamine and cancer: Cell biology, physiology, and clinical opportunities. J Clin Invest 123: 3678-3684, 2013.

4. Ferro M, Terracciano D, Buonerba C, Lucarelli G, Bottero D, Perdonà S, Autorino R, Serino $\mathrm{A}$, Cantiello F, Damiano R, et al: The emerging role of obesity, diet and lipid metabolism in prostate cancer. Future Oncol 13: 285-293, 2016.

5. Hilvo M, Denkert C, Lehtinen L, Müller B, Brockmöller S, Seppänen-Laakso T, Budczies J, Bucher E, Yetukuri L, Castillo S, et al: Novel theranostic opportunities offered by characterization of altered membrane lipid metabolism in breast cancer progression. Cancer Res 71: 3236-3245, 2011.

6. Currie E, Schulze A, Zechner R, Walther TC and Farese RV Jr: Cellular fatty acid metabolism and cancer. Cell Metab 18: 153-161, 2013.

7. Long JP, Li XN and Zhang F: Targeting metabolism in breast cancer: How far we can go? World J Clin Oncol 7: 122-130, 2016.

8. Trezzi JP, Vlassis N and Hiller K: The role of metabolomics in the study of cancer biomarkers and in the development of diagnostic tools. Adv Exp Med Biol 867: 41-57, 2015.

9. Siegel RL, Miller KD and Jemal A: Cancer statistics, 2016. CA Cancer J Clin 66: 7-30, 2016. 
10. Bauer KR, Brown M, Cress RD, Parise CA and Caggiano V: Descriptive analysis of estrogen receptor (ER)-negative, progesterone receptor (PR)-negative, and HER2-negative invasive breast cancer, the so-called triple-negative phenotype: A population-based study from the California cancer Registry. Cancer 109: 1721-1728, 2007.

11. Nielsen TO, Hsu FD, Jensen K, Cheang M, Karaca G, Hu Z, Hernandez-Boussard T, Livasy C, Cowan D, Dressler L, et al: Immunohistochemical and clinical characterization of the basallike subtype of invasive breast carcinoma. Clin Cancer Res 10: 5367-5374, 2004.

12. Choi J, Jung WH and Koo JS: Metabolism-related proteins are differentially expressed according to the molecular subtype of invasive breast cancer defined by surrogate immunohistochemistry. Pathobiology 80: 41-52, 2013.

13. Kim S, Kim DH, Jung WH and Koo JS: Expression of glutamine metabolism-related proteins according to molecular subtype of breast cancer. Endocr Relat Cancer 20: 339-348, 2013.

14. Kim S, Lee Y and Koo JS: Differential expression of lipid metabolism-related proteins in different breast cancer subtypes. PLoS One 10: e0119473, 2015.

15. Wang YY, Kuhajda FP, Li J, Finch TT, Cheng P, Koh C, Li T, Sokoll LJ and Chan DW: Fatty acid synthase as a tumor marker: Its extracellular expression in human breast cancer. J Exp Ther Oncol 4: 101-110, 2004.

16. Vazquez-Martin A, Ortega-Delgado FJ, Fernandez-Real JM and Menendez JA: The tyrosine kinase receptor HER2 (erbB-2): From oncogenesis to adipogenesis. J Cell Biochem 105: 1147-1152, 2008.

17. Zhou W, Simpson PJ, McFadden JM, Townsend CA, Medghalchi SM, Vadlamudi A, Pinn ML, Ronnett GV and Kuhajda FP: Fatty acid synthase inhibition triggers apoptosis during S phase in human cancer cells. Cancer Res 63: 7330-7337, 2003.

18. Menendez JA, Vellon L, Mehmi I, Oza BP, Ropero S, Colomer R and Lupu R: Inhibition of fatty acid synthase (FAS) suppresses HER2/neu (erbB-2) oncogene overexpression in cancer cells. Proc Natl Acad Sci USA 101: 10715-10720, 2004.

19. Menendez JA, Vellon L, Colomer R and Lupu R: Pharmacological and small interference RNA-mediated inhibition of breast cancer-associated fatty acid synthase (oncogenic antigen-519) synergistically enhances Taxol (paclitaxel)-induced cytotoxicity. Int J Cancer 115: 19-35, 2005.

20. Vazquez-Martin A, Ropero S, Brunet J, Colomer R and Menendez JA: Inhibition of fatty acid synthase (FASN) synergistically enhances the efficacy of 5-fluorouracil in breast carcinoma cells. Oncol Rep 18: 973-980, 2007.

21. Soupene E and Kuypers FA: Mammalian long-chain acyl-CoA synthetases. Exp Biol Med (Maywood) 233: 507-521, 2008.

22. Coleman RA and Lee DP: Enzymes of triacylglycerol synthesis and their regulation. Prog Lipid Res 43: 134-176, 2004.

23. Soupene E, Fyrst H and Kuypers FA: Mammalian acylCoA:lysophosphatidylcholine acyltransferase enzymes. Proc Natl Acad Sci USA 105: 88-93, 2008.

24. Park JH, Vithayathil S, Kumar S, Sung PL, Dobrolecki LE, Putluri V, Bhat VB, Bhowmik SK, Gupta V, Arora K, et al: Fatty acid oxidation-driven Src links mitochondrial energy reprogramming and oncogenic properties in triple-negative breast cancer. Cell Rep 14: 2154-2165, 2016.

25. Pucci S, Zonetti MJ, Fisco T, Polidoro C, Bocchinfuso G, Palleschi A, Novelli G, Spagnoli LG and Mazzarelli P: Carnitine palmitoyl transferase-1A (CPT1A): A new tumor specific target in human breast cancer. Oncotarget 7: 19982-19996, 2016.

26. Monaco ME, Creighton CJ, Lee P, Zou X, Topham MK and Stafforini DM: Expression of long-chain fatty acyl-CoA synthetase 4 in Breast and prostate cancers is associated with sex steroid hormone receptor negativity. Transl Oncol 3: 91-98, 2010.

27. Wu X, Li Y, Wang J, Wen X, Marcus MT, Daniels G, Zhang DY, Ye F, Wang LH, Du X, et al: Long chain fatty Acyl-CoA synthetase 4 is a biomarker for and mediator of hormone resistance in human breast cancer. PLoS One 8: e77060, 2013.

28. Cerami E, Gao J, Dogrusoz U, Gross BE, Sumer SO, Aksoy BA, Jacobsen A, Byrne CJ, Heuer ML, Larsson E, et al: The cBio cancer genomics portal: An open platform for exploring multidimensional cancer genomics data. Cancer Discov 2: 401-404, 2012.

29. Gao J, Aksoy BA, Dogrusoz U, Dresdner G, Gross B, Sumer SO, Sun Y, Jacobsen A, Sinha R, Larsson E, et al: Integrative analysis of complex cancer genomics and clinical profiles using the cBioPortal. Sci Signal 6: pl1, 2013.
30. Kao KJ, Chang KM, Hsu HC and Huang AT: Correlation of microarray-based breast cancer molecular subtypes and clinical outcomes: Implications for treatment optimization. BMC Cancer 11: 143,2011

31. Itoh M, Iwamoto T, Matsuoka J, Nogami T, Motoki T, Shien T, Taira N, Niikura N, Hayashi N, Ohtani S, et al: Estrogen receptor (ER) mRNA expression and molecular subtype distribution in ER-negative/progesterone receptor-positive breast cancers. Breast Cancer Res Treat 143: 403-409, 2014.

32. Minn AJ, Gupta GP, Siegel PM, Bos PD, Shu W, Giri DD, Viale A, Olshen AB, Gerald WL and Massagué J: Genes that mediate breast cancer metastasis to lung. Nature 436: 518-524, 2005.

33. Miyake T, Nakayama T, Naoi Y, Yamamoto N, Otani Y, Kim SJ, Shimazu K, Shimomura A, Maruyama N, Tamaki Y, et al: GSTP1 expression predicts poor pathological complete response to neoadjuvant chemotherapy in ER-negative breast cancer. Cancer Sci 103: 913-920, 2012.

34. van 't Veer LJ, Dai H, van de Vijver MJ, He YD, Hart AA, Mao M, Peterse HL, van der Kooy K, Marton MJ, Witteveen AT, et al: Gene expression profiling predicts clinical outcome of breast cancer. Nature 415: 530-536, 2002.

35. Wang Y, Klijn JG, Zhang Y, Sieuwerts AM, Look MP, Yang F, Talantov D, Timmermans M, Meijer-van Gelder ME and Yu J: Gene-expression profiles to predict distant metastasis of lymphnode-negative primary breast cancer. Lancet 365: 671-679, 2005.

36. Barretina J, Caponigro G, Stransky N, Venkatesan K, Margolin AA, Kim S, Wilson CJ, Lehár J, Kryukov GV, Sonkin D, et al: The Cancer Cell Line Encyclopedia enables predictive modelling of anticancer drug sensitivity. Nature 483: 603-607, 2012.

37. Györffy B, Lanczky A, Eklund AC, Denkert C, Budczies J, Li Q and Szallasi Z: An online survival analysis tool to rapidly assess the effect of 22,277 genes on breast cancer prognosis using microarray data of 1,809 patients. Breast Cancer Res Treat 123: 725-731, 2010

38. Cho CY, Lee KT, Chen WC, Wang CY, Chang YS, Huang HL, Hsu HP, Yen MC, Lai MZ and Lai MD: MST3 promotes proliferation and tumorigenicity through the VAV2/Rac1 signal axis in breast cancer. Oncotarget 7: 14586-14604, 2016.

39. Golej DL, Askari B, Kramer F, Barnhart S, Vivekanandan-Giri A, Pennathur S and Bornfeldt KE: Barnhart S, VivekanandanGiri A, Pennathur S and Bornfeldt KE: Long-chain acyl-CoA synthetase 4 modulates prostaglandin E2 release from human arterial smooth muscle cells. J Lipid Res 52: 782-793, 2011.

40. Subik K, Lee JF, Baxter L, Strzepek T, Costello D, Crowley P, Xing L, Hung MC, Bonfiglio T, Hicks DG, et al: The expression patterns of ER, PR, HER2, CK5/6, EGFR, Ki-67 and AR by immunohistochemical analysis in breast cancer cell lines. Breast Cancer (Auckl) 4: 35-41, 2010.

41. Kaemmerer E, Peuscher A, Reinartz A, Liedtke C, Weiskirchen R, Kopitz J and Gassler N: Human intestinal acyl-CoA synthetase 5 is sensitive to the inhibitor triacsin C. World J Gastroenterol 17: 4883-4889, 2011.

42. Vessey DA, Kelley M and Warren RS: Characterization of triacsin C inhibition of short-, medium-, and long-chain fatty acid: CoA ligases of human liver. J Biochem Mol Toxicol 18: 100-106, 2004

43. Soltysiak RM, Matsuura F, Bloomer D and Sweeley CC: D,L-alpha-Fluoropalmitic acid inhibits sphingosine base formation and accumulates in membrane lipids of cultured mammalian cells. Biochim Biophys Acta 792: 214-226, 1984.

44. Askari B, Kanter JE, Sherrid AM, Golej DL, Bender AT, Liu J, Hsueh WA, Beavo JA, Coleman RA and Bornfeldt KE: Rosiglitazone inhibits acyl-CoA synthetase activity and fatty acid partitioning to diacylglycerol and triacylglycerol via a peroxisome proliferator-activated receptor-gamma-independent mechanism in human arterial smooth muscle cells and macrophages. Diabetes 56: 1143-1152, 2007.

45. Chen WC, Wang CY, Hung YH, Weng TY, Yen MC and Lai MD: Systematic analysis of gene expression alterations and clinical outcomes for long-chain acyl-coenzyme A synthetase family in cancer. PLoS One 11: e0155660, 2016.

46. Orlando UD, Castillo AF, Dattilo MA, Solano AR, Maloberti PM and Podesta EJ: Acyl-CoA synthetase-4, a new regulator of mTOR and a potential therapeutic target for enhanced estrogen receptor function in receptor-positive and -negative breast cancer. Oncotarget 6: 42632-42650, 2015. 
47. Mody M, Dharker N, Bloomston M, Wang PS, Chou FS, Glickman TS, McCaffrey T, Yang Z, Pumfery A, Lee D, et al: Rosiglitazone sensitizes MDA-MB-231 breast cancer cells to anti-tumour effects of tumour necrosis factor-alpha, $\mathrm{CH} 11$ and CYC202. Endocr Relat Cancer 14: 305-315, 2007.

48. Doll S, Proneth B, Tyurina YY, Panzilius E, Kobayashi S, Ingold I, Irmler M, Beckers J, Aichler M, Walch A, et al: ACSL4 dictates ferroptosis sensitivity by shaping cellular lipid composition. Nat Chem Biol 13: 91-98, 2017.

49. Khurana P, Gokhale RS and Mohanty D: Genome scale prediction of substrate specificity for acyl adenylate superfamily of enzymes based on active site residue profiles. BMC Bioinformatics 11: 57 , 2010.

50. Kanter JE, Tang C, Oram JF and Bornfeldt KE: Acyl-CoA synthetase 1 is required for oleate and linoleate mediated inhibition of cholesterol efflux through ATP-binding cassette transporter A1 in macrophages. Biochim Biophys Acta 1821: 358-364, 2012.

51. Lopes-Marques M, Cunha I, Reis-Henriques MA, Santos MM and Castro LF: Diversity and history of the long-chain acyl-CoA synthetase (Acsl) gene family in vertebrates. BMC Evol Biol 13: $271,2013$.
52. Bu SY and Mashek DG: Hepatic long-chain acyl-CoA synthetase 5 mediates fatty acid channeling between anabolic and catabolic pathways. J Lipid Res 51: 3270-3280, 2010.

53. Gassler N, Roth W, Funke B, Schneider A, Herzog F, Tischendorf JJ, Grund K, Penzel R, Bravo IG, Mariadason J, et al: Regulation of enterocyte apoptosis by acyl-CoA synthetase 5 splicing. Gastroenterology 133: 587-598, 2007.

54. Röhrig F and Schulze A: The multifaceted roles of fatty acid synthesis in cancer. Nat Rev Cancer 16: 732-749, 2016.

55. Igal RA, Wang P and Coleman RA: Triacsin C blocks de novo synthesis of glycerolipids and cholesterol esters but not recycling of fatty acid into phospholipid: Evidence for functionally separate pools of acyl-CoA. Biochem J 324: 529-534, 1997.

56. Sastre-Serra J, Nadal-Serrano M, Pons DG, Valle A, Oliver J and Roca P: The effects of $17 \beta$-estradiol on mitochondrial biogenesis and function in breast cancer cell lines are dependent on the ER $\alpha /$ ER $\beta$ ratio. Cell Physiol Biochem 29: 261-268, 2012.

57. Zhou Z, Zhou J and Du Y: Estrogen receptor beta interacts and colocalizes with HADHB in mitochondria. Biochem Biophys Res Commun 427: 305-308, 2012. 